\title{
Problèmes de contrôle biologique des Insectes vecteurs
}

\author{
Par Jaroslav WEISER
}

Les recherches de ces dernières années ont révélé de nouveaux aspects de la lutte contre les Insectes vecteurs des maladies humaines et des animaux domestiques. De plus en plus, on a pu montrer que des quantités considérables d'insecticides persistent dans les régions traitées par ceux-ci et constituent par leur présence dans le corps des animaux un danger constant pour l'homme. Dans d'autres cas, c'est la résistance des Arthropodes vecteurs aux insecticides qui nous oblige à chercher de nouvelles techniques de lutte pour les grandes campagnes antipaludiques. On a observé des foyers locaux, où les vecteurs sont si cachés et si dispersés que ce sont seulement les méthodes biologiques qui sont capables de réduire leur nombre en dessous du nombre nécessaire au maintien de l'endémie. Aussi, c'est aux méthodes biologiques que l'on fait de plus en plus confiance.

Quels sont les facteurs biologiques utilisables dans la lutte contre les vecteurs des maladies ? En général, il s'agit des entomophages (parasites et prédateurs) et des maladies d'Insectes. La plupart des prédateurs ne sont pas spécifiques: ils attaquent leur proie immédiatement, la tuent sans qu'il se déroule de processus embryonnaire dans le corps du vecteur. Au contraire, les parasites déposent leurs œufs dans les tissus du vecteur à divers stades (œuf, chenille ou larve, pupe ou adulte) ; les larves se développent dans le corps de l'hôte. Quand le parasite de l'Arthropode a atteint sa maturité, il quitte son hôte qui meurt alors, pour en chercher un autre. La plupart des espèces parasites s'attaquent à des formes terrestres, alors que les prédateurs sont présents aussi bien dans les biotopes terrestres que aquatiques.

Pour pouvoir utiliser ces auxiliaires, on doit les élever, dans la plupart des cas, dans un insectarium, sur les hôtes convenables. On ne peut les conserver vivants pendant longtemps; on les utilise pour des colonisations locales ou des introductions en masse dans les régions où ils ne sont pas présents.

Les maladies sont des facteurs différents. Elles se répandent par contact entre les individus infectés et les sains ; plus les contacts sont fréquents et importants, mieux se fait la transmission. Celle-ci dépend donc des degrés de contact des populations ; dans la plupart des cas, la contamination produit chez les Insectes des épizooties ou des panzooties massives. Chez les Arthropodes vecteurs, on trouve les mêmes groupes de maladies que chez les Vertébrés - virus, rickettsies, mycoses —, Bactéries, Protozoaires et Nématodes. La plupart des agents de ces infections sont spécifiques, non pathogènes et non toxiques pour les Vertébrés. On peut cultiver en masse sur des milieux artificiels 
la plupart des Bactéries, des Champignons et quelques Nématodes. Pour les autres, il faut les faire se développer sur les hôtes dans des élevages. La plupart d'entre eux donnent des spores ou des stades définitifs qu'on peut conserver infectants pendant longtemps, dans quelques cas pendant plus de dix ans.

On connaît de nombreuses maladies et espèces d'entomophages s'attaquant aux différents groupes de vecteurs de maladies, mais on ne sait pas beaucoup sur la distribution de ces facteurs de limitation dans la nature. En voici quelques exemples:

\section{Chez les Blattoïdés.}

Chez les Blattes, il existe un groupe de parasites spécialisés dans le parasitisme des œufs : ils sont plus actifs dans les pays tropicaux, mais ont été trouvés en Europe. Les genres Prosevania et Evania attaquent les Blatta, Blatella et Periplaneta et on peut les cultiver au laboratoire en élevage de Blattes. A part d'une maladie douteuse signalée par Grégoire (1951), nous ne connaissons pas de virus chez les Blattes. Mais quelques expériences avec une rickettsie des Grillons, Rickettsiella grylli Vago et Martoja 1963, ont montré qu'il serait aussi possible d'utiliser un organisme semblable contre les Blattes. Les habitudes de cannibalisme de ces animaux favorisent l'extension des maladies infectieuses. Une affection bactérienne de Blatta orientalis a été découverte par Heinecke (1956), mais une souche isolée dans ce cas a perdu sa virulence après quelques passages sur milieu artificiel. Quelques Sporozoaires sont présents dans les tissus des Blattes. Coelosporidium periplanetae Schwarzewski 1914 dans les tubes de Malpighi, Plistophora kudoi Sprague et Ramsay 1943 dans la paroi intestinale, Plistophora blattae Weiser 1961 dans le corps adipeux et Plistophora periplanetae Lutz et Splendore 1904 dans les tubes de Malpighi chez Blatta orientalis.

Une Coccidie, Adelina cryptocerci Yearwood 1937, est trouvée chez une Blatte phytophage, Cryptocercus punctulatus, dans le corps adipeux. Dans les conditions expérimentales, Welch a réussi à infecter des Blattes avec des larves du Nématode DD 136 (Neoaplectana sp. de Dutky). C'est un représentant d'un groupe de Nématodes qui constituent de véritables agents mécaniques inoculateurs de Bactéries. Celles-ci constituent une nourriture normale pour ces Nématodes qui les transportent toute leur vie dans leur œsophage. Quand les larves du Nématode pénètrent dans le corps de l'Insecte, les Bactéries alors s'y développent, causant une septicémie et tuant l'hôte. Celui-ci meurt après 24 à $48 \mathrm{~h}$ de contact et dans son corps se développent deux générations successives du Nématode. Quand la nourriture est épuisée, les larves du Nématode quittent alors l'hôte et en cherchent un autre.

\section{Chez les Anoploures.}

Les Poux n'hébergent pas de maladies. Cependant, les rickettsies qu'ils transmettent attaquent leurs cellules épithéliales et raccourcissent leur vie. Dans quelques cas même, on peut observer des animaux rougeâtres ; c'est que leur intestin est rompu et que l'hémoglobine du sang qu'ils ont ingéré s'est distribuée dans l'hémolymphe. Dans 
un cas, Popov et Manuilova (1926) ont trouvé une Microsporidie, Nosema pediculi, dans la paroi intestinale de Pediculus humanus corporis en Russie.

\section{Chez les Hétéroptères.}

Les Punaises et les Triatomes ne sont pas bien étudiés à ce point de vue. Dans les œufs des Hétéroptères, on a trouvé quelques parasites, parmi lesquels Telenomus fariasi, qui s'observe chez les espèces exoanthropes en Europe et chez les Triatomes synanthropes en Amérique. Jusqu'à 16 parasites peuvent se développer dans un œuf parasité. Grâce aux recherches de Poisson (1924), nous connaissons quelques Microsporidies des Punaises aquatiques. Dans Cimex rotundatus, Shortt et Swaminath (1924) ont trouvé aux Indes une autre espèce, Nosema adiei. Cette Microsporidie attaque les tissus des Punaises y compris l'ovaire. Deux Schizogrégarines, Machadoella spinigeri (Machado, 1913 ) et $M$. triatomae (Reichenow, 1935) détruisent les cellules des tubes de Malpighi.

\section{Chez les Aphaniptères.}

Les Puces n'hébergent, dans l'état des connaissances actuelles, que quelques maladies. On ne leur connaît pas de parasites Hyménoptères. Seuls, les Pseudoscorpionidés et les Chenilles de Tineola biselliella sont connus comme prédateurs des larves de Puces. Quand Nöller (1914) a étudié la transmission des Trypanosomes par des Puces, il a trouvé dans les élevages de Ceratophyllus gallinae et de $C$. columbae une Coccidie, Legerella parva. Une autre, L. grassi, a été signalée par Splendore dans les tubes de Malpighi de Nosopsyllus fasciatus. Dans des élevages de Ctenocephalides canis et de C. felis, Nöller (1912) a également trouvé une Microsporidie, Nosema pulicis, attaquant les organes des larves et aussi les ovaires et les œufs. Les larves sont infectées dans les nids par les déjections de Puces. La mortalité dans les cas étudiés était spectaculaire. Une autre espèce, Nosema ctenocephali, a été trouvée par Korke dans Ctenocephalides felis. Une troisième souche fut observée également chez Archaeopsylla erinacei (Weiser 1957).

\section{Chez les Diptères.}

Les Diptères constituent un groupe d'organismes si divers sous beaucoup de leurs aspects qu'il est nécessaire de les étudier ici famille par famille. Les Moustiques sont attaqués par de nombreux prédateurs qui réduisent la quantité de leurs larves dans les marais. Les Grenouilles, les Poissons, les larves de Trichoptères, Coléoptères, Hémiptères, Odonates et même les Diptères comme Chaoborus ou Toxorhynchites sont des ennemis considérables des larves de Moustiques dans la nature. Une grande difficulté se présente dans tous les programmes de contrôle des larves de Culicidés par introduction massive des prédateurs. C'est que la plupart des Moustiques ont un cycle larvaire d'environ 4 semaines seulement au printemps. Après, les prédateurs ne trouvent plus de nourriture, leurs gîtes se dessèchent en effet chaque année et ils doivent, pour pouvoir se maintenir, avoir à leur disposition des biotopes constitués par des 
collections d'eau permanentes. L'action des maladies des Moustiques est plus marquée : ces maladies persistent dans les gîtes larvaires même quand leurs hôtes en sont disparus et l'eau évaporée. Il est possible de retrouver les agents des mêmes maladies dans les mêmes biotopes pendant des dizaines d'années. Trois groupes de maladies n'ont qu'une influence problématique sur les Moustiques: les virus, les rickettsies et les bactéries. Tout ce que nous connaissons de ces groupes concerne les Chironomidés et les Tipulidés. Des virus avec différentes inclusions protéiniques sont connus chez Tipula paludosa (Smith et Xéros, 1954) et chez Camptochironomus tentans (Weiser, 1948) au niveau du corps adipeux. La présence d'un autre virus, moins spécifique, le «virus iridescent » des Tipula (Smith, Hills, Rivers 1961), a pu être démontrée chez plus de 20 hôtes secondaires. Dans un cas, Kellen et coll. ont signalé (1963) des corpuscules polyhédriques analogues aux inclusions de polyhédrose chez Culex tarsalis en Californie. Des Rickettsies ont été également trouvées dans le corps adipeux de Camptochironomus tentans (Weiser, 1948) et de Tipula paludosa (Müller-Kögler, 1958); les larves infectées sont d'une blancheur de porcelaine. L'infection, plus spécifique pour l'hôte que celle par virus, est transmise par la nourriture contaminée. Les Moustiques n'hébergent pas de Bactéries pathogènes spécifiques. Dans quelques cas, les souches de Bacillus thuringiensis, qui produisent une toxine soluble thermostable, sont capables de les tuer. Mais les quantités nécessaires sont trop élevées pour une utilisation dans la nature.

De grande importance sont les mycoses causées par les Coelomomyces. Ces Blastocladiales présentent des sporanges lisses ou cannelés, ovalaires. Plus d'une vingtaine d'espèces sont connues de toutes les parties du monde. En Europe, on a trouvé Coelomomyces psorophorae chez les larves de Aedes vexans en Tchécoslovaquie (Weiser et Vavra) et $C$. raffaelei chez les larves de Anopheles claviger en Italie (Coluzzi et Rioux, 1962). Dans les régions tropicales, si l'on a parfois trouvé jusqu'à 60 à $80 \%$ d'infections, dans la plupart des cas, les pourcentages s'élèvent seulement de 5 à $10 \%$. Les sporanges sont disséminés par les Moustiques adultes porteurs d'une infection latente. Laird (1959) a montré qu'il est possible d'introduire, en utilisant des sporanges, Coelomomyces stegomyiae dans une région où la maladie est absente.

Un autre groupe de grande importance pour les Moustiques sont les Entomophthorées: Entomophthora gracilis, E. montana, E. papillata, E. apiculata, E. culicis et $E$. conglomerata (Thaxter, 1888) sont les espèces qui ont été signalées chez les Moustiques. La plupart sont des parasites non spécifiques qui attaquent les Insectes affaiblis après la ponte. Cependant, dans d'autres cas, Weiser et Novak (1962) ont trouvé de nombreux Moustiques hibernants attaqués et détruits par le Champignon dans leurs retraites d'hiver. C'est Entomophthora conglomerata qui a été isolée en culture de ces Insectes. Quelques Entomophthorées sont cultivables sur les milieux artificiels et il est possible d'en produire les spores en masse. Les méthodes d'utilisation de ces spores font l'objet d'études.

Les maladies des Moustiques causées par les Protozoaires sont bien connues. L'étude en effet en a été faite en connection avec celle de la transmission du paludisme. Dans la plupart des cas, ces Protozoaires apparaissent sous forme de kystes blanchâtres dans le corps adipeux. Une Grégarine, parasite transitoire, Lankesteria culicis, attaque 
l'intestin des larves arboricoles de Aedes geniculatus. Mais la plupart des cellules détruites par la Grégarine sont remplacées par régénération. Une espèce plus pathogène, causant plus de destructions, est une Schizogrégarine, Caulleryella pipientis, vivant dans l'intestin de Culex pipiens en Europe (Bresslau et Buschkiel, 1919).

Une longue liste de Microsporidies est connue chez les larves des Moustiques. La plupart sont des parasites du corps adipeux, comme Thelohania opacita chez les larves de Aëdes vexans, A. sticticus, A. annulipes, Culex territans et $C$. apicalis, ou Thelohania legeri chez Anopheles maculipennis, A. bifurcatus, A. punctipennis, A. quadrimaculatus et $A$. crucians. Plus de 15 espèces sont signalées des Moustiques d'Amérique du Nord. Quelques-unes d'entre elles sont transmises dans les œufs d'une génération à l'autre. Ce processus a été observé pour Nosema stegomyiae, Thelohania grassi, Plistophora collesi, Thelohania californica ou $T$. benigna (voir Weiser, 1961). Toutes les tentatives pour infecter des larves de Moustiques au laboratoire par les Microsporidies sont restées vaines jusqu'à maintenant. Dans d'autres cas cependant, pour Plistophora culicis ou Nosema stegomyiae, on a réussi à infecter les Moustiques adultes en leur donnant comme nourriture de l'eau sucrée contaminée (Canning, 1957, Fox et Weiser, 1959). Ces expérimentations nous ouvrent la perspective de l'utilisation possible des Microsporidies dans les conditions naturelles.

Enfin, il existe aussi des Nématodes qui attaquent les larves de Moustiques. Dans quelques endroits, on trouve une infection par Mermitidés. Hydromermis churchillensis et quelques autres espèces sont parasites de Aëdes communis (Welch, 1958); dans la plupart des gîtes larvaires, on ne trouve que 3 à $5 \%$ des larves parasitées ; dans quelques cas, toutes les larves le sont. Il convient donc de rechercher les conditions nécessaires à une infection massive des larves. Chez les Moustiques s'observent seulement les larves de Mermitidés. Celles-ci quittent leur hôte pour finir leur développement et devenir adultes dans le sable au fond des gîtes. Après que les larves des Mermitidés ont quitté leur hôte, celui-ci est tué par les Bactéries qui pénètrent dans son corps.

Les Simulidés, les Culicoïdes et Ceratopogon sont affectés par d'autres maladies, mais la plupart de celles-ci atteignent les stades larvaires, alors que les maladies des adultes sont presque inconnues. Chez les Simulidés, les mycoses sont représentées par Coelomycidium simulii. Les larves parasitées renferment alors plusieurs milliers de sphères blanchâtres contenant une grande quantité de zoospores flagellées. Pendant la nymphose, les larves meurent et les zoospores sont libérées dans l'eau. Dans les foyers naturels, l'infection se présente chez 5 à $40 \%$ des larves pendant l'été et le printemps. Chez les Simulidés, on trouve également plus de neuf espèces de Microsporidies. Quelques-unes de celles-ci n'infestent les Simulidés que pendant une saison. Nosema stricklandi et Caudospora simulii (Weiser, 1961) sont les agents d'infections de printemps ; Plistophora simulii, Thelohania fibrata, $T$. bracteata et $T$. varians infestent les populations d'été. De 15 à $20 \%$ seulement des larves sont infectées pendant les premiers jours qui suivent l'éclosion des œufs. Chez les Simulidés, on trouve aussi des Mermitidés qui parasitent parfois plus de $80 \%$ des larves, dans la plupart des cas au printemps.

Chez les Cératopogonidés, on a trouvé une Schizogrégarine, Schizocystis gregarinoides, chez Ceratopogon solstitialis, et plusieurs Microsporidies, Nosema sphaeromya- 
dis, Toxoglugea vibrio et Spiroglugea octosporea. Mais nos connaissances actuelles des maladies des Ceratopogon et Culicoïdes sont très fragmentaires.

Les Mouches, comme animaux terrestres, ont aussi plusieurs parasites spécifiques. La plupart de ceux-ci sont connus à la suite des études faites sur la Mouche tsé-tsé. Plus de 20 espèces d'Hyménoptères ont été signalées s'attaquant à Glossina palpalis. Dans un cas, celui de Syntomophyrum glossinae, on a pu effectuer des élevages en masse et lâcher les parasites dans les forêts à Glossines. Cela eut comme résultat d'augmenter la fréquence des infestations pendant quelques semaines mais, plus tard, tout revint à l'état originel. Chez les Glossines, nous ne connaissons pas de maladies spécifiques, sauf un organisme douteux, Myxosporidium heibergi Dutton, Todd et Tobey (1907). Ce Sporozoaire, probablement une Microsporidie, était présent dans la paroi intestinale de Glossina palpalis. Dans certains cas, Hepatozoon pettiti forme chez les Glossines des kystes globuleux qui remplissent leur cavité générale.

Les Mouches ont leurs œufs attaqués par des Hyménoptères parasites. Ainsi, Polystes hebraeus, importé aux îles Fiji, a réduit le nombre des Mouches domestiques dans les îles traitées. Les Spalangia ont une action effective contre d'autres espèces dans les régions subtropicales et tropicales de l'Amérique. Utilisées dans les îles du Pacifique et à Porto-Rico, elles ont produit un bon effet et une réduction des populations de Mouches domestiques.

Les maladies des Mouches, particulièrement des larves, ne sont pas bien connues. Celles-ci hébergent des microorganismes, Bactéries, Protozoaires, Champignons, peutêtre aussi des virus (virus iridescent) et des Rickettsies; mais la mise en évidence de ces maladies dans la nature est difficile, car les larves libres ne sont pas étudiées. Seulement deux noms, Bacillus lutzae et Staphylococcus muscae, rappellent deux cas de maladies des larves. Ces dernières années, Dunn (1960) et Briggs (1960) ont réussi à réduire le nombre des Mouches dans la nature et dans les élevages en ajoutant Bacillus thuringiensis à la nourriture des poules. Dans quelques cas, nous avons observé que Proteus morgani était un facteur provoquant une haute mortalité des larves dans les élevages de laboratoire. Dans plusieurs cas, on a trouvé des Microsporidies causant des épizooties mortelles. Octosporea muscae domesticae Flu 1911 est l'agent d'une maladie des tubes de Malpighi et de l'intestin postérieur chez Musca domestica, et aussi chez Drosophila confusa et $D$. plurilineata. On les a trouvées aussi dans les œufs des hôtes. Par cette voie, l'infection est transmise d'une génération à l'autre. Des espèces voisines de Microsporidies ont été trouvées dans d'autres espèces-hôtes: Octosporea monospora, Thelohania thomsoni (Kramer, 1961) et quelques autres. Fantham et Porter (1958) ont étudié les maladies des larves de Calliphora et Musca dans des élevages massifs destinés aux pêcheurs et ils y ont signalé une haute mortalité.

Des épizooties spectaculaires chez les Mouches sont causées chaque année par les Entomophthoracées. Dans la plupart des cas, c'est Entomophthora muscae, mais, dans d'autres cas, on a déterminé E. calliphorae, E. americana ou E. sphaerosperma. Il existe des foyers locaux d'où se répartissent dans les zones environnantes les Mouches infectées. Dans quelques laboratoires (Baird, 1957), l'infection a pu être maintenue dans les élevages de Mouches ou de Tachinaires pendant quelques semaines, mais seulement 
dans un insectarium, avec des conditions spéciales d'élevage. Nous avons aussi quelques expériences où les spores de Beauveria bassiana ont tué des Mouches domestiques.

Nous ne connaissons pas de maladie par microorganismes chez les Tabanidés, et ce sont seulement les parasites comme Phanurus emersoni ou les Telenomus qui participent à la réduction du nombre de ces Insectes piqueurs. Au Texas, Phanurus emersoni a provoqué une réduction de $50 \%$ des Tabanus dans la région mise en expérimentation.

Les moins connus sont les maladies et les parasites des Phlébotomes. On ne connaît qu'une Entomophthora, E. papatassi, signalée chez les Phlébotomes dans la région méditerranéenne.

Dans cette discussion, nous ne devons pas oublier les autres vecteurs de maladies, les Acariens et les Mollusques.

\section{Chez les Acariens.}

Nous-même avons déjà signalé les Microsporidies Nosema steinhausi chez Tyrophagus noxius, Nosema ixodis chez Ixodes ricinus (Weiser 1957). Quelques autres espèces parasites sont signalées chez les Opilionidés et Hydracariens. Rehácek a trouvé une septicémie des Tiques causée par Proteus mirabilis chez Rhipicephalus sanguineus. Dans la nature, Ixodes ricinus est attaqué par Beauveria bassiana; on a aussi trouvé trois à quatre espèces d'Hyménoptères parasites de Tiques. Avec l'un d'eux, Hunterellus hookeri, on a essayé dans quelques localités de réduire les populations de la Tique Dermacentor variabilis, mais sans succès (Smith et Cole, 1943).

\section{Chez les Mollusques.}

Chez les Mollusques hôtes intermédiaires d'Helminthes, on trouve des maladies par Protozoaires (Haplosporidia, Microsporidia), des parasites et des prédateurs. Comme cela a été démontré par Barrow (1960), la détection des maladies est seulement une question de dissection d'une quantité adéquate de Mollusques. C'est la même chose pour Plistophora husseyi chez Physa heterostropha et Aplexa hypnorum (Michelson, 1963).

Un grand rôle dans la réduction des Mollusques vecteurs est joué par les Mollusques prédateurs, représentés par exemple par Marisa cornuarietis. Cette espèce a exercé, associée aux Molluscocides, un grand effet sur les populations d'Australorbis glabrata dans l'île de Viéques, à Porto-Rico. Un grand nombre de ces prédateurs a été signalé par Simmonds (1960). Berg (1962) a montré que plus de 58 espèces de Schizomyzidae (Diptères) sont parasites des Mollusques hôtes intermédiaires de Trématodes. Des essais d'introduction de ces Mouches dans certains groupes d'îles du Pacifique ont commencé.

Nous voyons donc que de nombreux parasites et maladies participent à la réduction des vecteurs. C'est maintenant à nous de préparer les conditions pour une production massive de ces agents et une réduction des vecteurs d'une façon économique. Les foyers d'infection se forment dans des endroits différents du monde. Dans beaucoup de cas, le fait de signaler le facteur, puis de l'isoler et de l'utiliser en laboratoire, sera le commencement des applications pratiques. 


\section{Bibliographie}

BAIRD (R. B.), 1957. - Notes on a laboratory infection of Diptera caused by the fungus Empusa muscae Cohn. Canad. Entomologist, 84, pp. 432-435.

BARRow̧ (J. H.), 1960. - Studies of Haplosporidium in fresh-water snails of Michigan. J. Protozool, 7, Suppl. 12.

Berg (C. O.), 1962. - Snail-killing sciomyzid flies: biology of the aquatic species. Proc. Int. Congres of Limnology, in press.

Bresslau (E.), Buschkiel (M.), 1919. - Parasiten der Stechmücken. Biol. Zentralbl., 39, pp. 325-336.

BRIGGS (J.), 1960. - Reduction of adult housefly emergence by the effects of Bacillus spp. on the development of immature forms. J. Insect. Pathol., 2, pp. 418-432.

Canning (E.), 1957. - On the occurence of Plistophora culicis Weiser in Anopheles gambiae. Riv. Malariol., 36, 1-3, pp. 39-50.

DuNN (P. H.), 1960. - Control of house flies in bovine feces by a feed additive containing Bacillus thuringiensis v. thuringiensis Berl. J. Insect. Pathol., 2, pp. 13-16.

Dutron (J. E.), Todp (J. L.), ToBey (J.), 1907. - Concerning certain parasitic protozoa observed in Africa. Ann. Trop. Med. Parasit., 1, pp. 287-370.

FANTHAM (H. B.), Porter (A.), 1958. - Some pathogenic bacteriform microsporidia from Crustacea and Insecta. Proc. Zool. Soc. London, 130, pp. 153-168.

Fox (W.), WeIser (J.), 1959. - A microsporidian parasite of Anopheles gambiae in Liberia. J. Parasitol., 45, pp. 21-30.

Gregorre (Ch.), 1951. - Virus-like bodies in the blood of the house cricket. J. Gen. Microbiol., 5, pp. 121-123.

HeINECKE (H.), 1956. - Ueber einen pathogenen Sporenbildner in der Haemolymphe von Blatta orientalis L. Zentr. Bact., II, 109, pp. 524-535.

Kellen (W. R.), Clark (T. B.), Lindegren (J. E.), 1963. - A possible polyhedrosis in Culex tarsalis Coqu. (Dipt., Culic.). J. Insect. Pathol., 5, pp. 98-103.

Kellen (W. R.), Wills (W.), 1962. - New Thelohania from Californian Mosquitoes (Nosematidae, Microsporidia). J. Insect. Pathol., 4, pp. 41-56.

KorKe (V. T.), 1916. - On a Nosema (Nosema pulicis n. sp.) parasitic in the dog flea (Ctenocephalus felis). Ind. J. Med. Res., 3, pp. 725-730.

KRAMER (J. P.), 1961. - Thelohania thomsoni n. sp., a Microsporidian parasite of Muscina assimilis (Fallén) (Dipt., Muscidae). J. Insect Pathol., 3, pp. 259-265.

Kudo (R. R.), 1924. - A biological and taxonomic study of the Microsporidia. 1ll. Biol. Monographs, 9, $268 \mathrm{p}$.

LAIRD (M.), 1959. - Fungal parasites of mosquito larvae from the Oriental and Australian regions, with a key to the genus Coelomomyces (Blastocladiales, Coelomomycetaceae). Canad. J. Zoology, 37, pp. 781-791.

Lutz (A.), Splendore (A.), 1904. - Ueber Pebrine und verwandte Mikrosporidien, II. Centr. Bact., 36, pp. 645-650.

MiCheLson (E. H.), 1963. - Plistophora husseyi sp. n., a microsporidian parasite of aquatic pulmonate snails. J. Insect Pathol., 5, pp. 28-38. 
MüLlER-KöGlER (E.), 1958. - Eine Rickettsiose der Tipula paludosa Larven. Naturwissensch., 45, p. 248.

NöLleR (W.), 1912. - Die Uebertragungsweise der Rattentrypanosomen durch Flöhe. Arch. Protistenk., 25, pp. 386-484.

Poisson (R.), 1929. - Recherches sur les Microsporidies parasites des Hémiptères. Arch. Zool. Exp. Gén., 69, pp. 55-63.

Poisson (R.), 1953. - Ordre des Microsporidies dans: Grassé, Traité de Zoologie, I, 2, pp. 1042-1070, Masson, Paris.

Popov (P.), Manullova (N.), 1926. - Obnarushenij mikrosporidii sp. nov. u vshej Pediculus vestimenti Nitzsch. Rus. Shurn. Trop. Med., 8, pp. 43-49.

Reichenow (E.), 1935. - Machadoella triatomae n. g. n. so., eine Schizogregarine aus Triatoma dimidiata. Zschr. Protistenk., 84, pp. 431-445.

REHáceK (J.), 1962, sous presse.

Simmonds (F. J.), 1961. - Report on a tour in Asia, May-October 1961. Commonw. Agr. Bureaux, 62 p.

Smrth (C. N.), Cole (M. M.), 1943. - Studies of parasites of the American Dog tick. J. Econ. Ent., 36, pp. 569-572.

Smith (K. M.), Hrils (G. J.), Rivers (C. F.), 1961. - Studies on the cross-inoculation of the Tipula iridescent virus. Virology, 13, pp. 233-241.

Smith (K. M.), Xeros (N.), 1954. - An unusual virus disease of a dipterous larva. Nature, 173, p. 866.

Sprague (V.), Ramsay (J.), 1941. - A preliminary note on Plistophora kudoi n. sp., a microsporidian parasite of the cockroach. Anat. Record, 81, pp. 132-133.

Steinhaus (E. A.), 1949. - Principles of Insect Pathology, McGraw-Hill, N.-York, 757 p.

Thaxter (R.), 1888. - The Entomophthoraceae of the United States. Mem. Boston Soc. Nat. Hist., 4, pp. 133-201.

Vago (C.), Martoja (R.), 1963. - Une rickettsiose chez les Gryllidae (Orthopt.). C.R. Acad. Sci., 256, pp. 1045-1057.

WeISER (J.), 1948. - Zwei neuartige Erkrankungen bei Insekten. Experientia, 4, pp. 317-318.

Weiser (J.), 1957. - Cizopasníci některych druhů hmyzu ssajících krev. Cisl. Parasitologie, 4, pp. 355-358.

WeISER (J.), 1961. - Die Mikrosporidien als Parasiten der Insekten. Monographien zur Angewandten Entomologie, 17, 149 p.

Weiser (J.), Novák (D.), 1962. - Auftreten von Mykosen bei Stechmücken. Colloque Int. sur la Pathol. d'Insectes, Paris.

Weiser (J.), VAVRA (J.), 1964. - Zur Verbreitung der Coelomomyces-Pilze in europäischen Insekten. Zschr. Tropenmed. u. Parasitol., 15, 38-42.

WeLCH (H. E.), 1958. - Test of a Nematode and its associated bacterium for control of the colorado potato beetle, Leptinotarsa decemlineata (Say). Ann. Rept., Ent. Soc. Ontario, 88, pp. 53-54.

YeARwood (E. A.), 1937. - The life cycle of Adelina cryptocerci, a coccidian parasite of the roach Cryptocercus punctulatus. Parasitol., 29, pp. 370-390.

(Laboratoire de Pathologie des Insectes, Académie des Sciences, Prague, Tchécoslovaquie) 